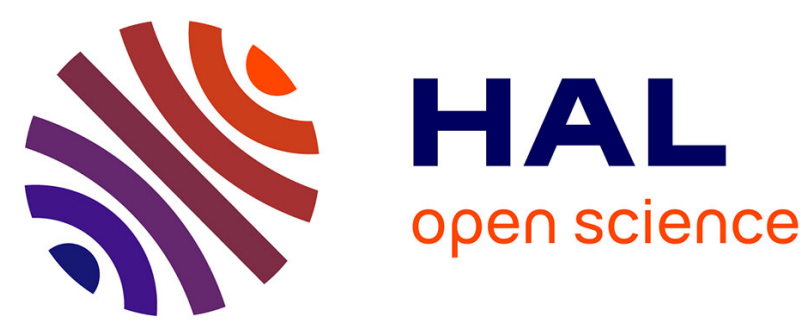

\title{
Antioxidant properties of tea blunt ROS-dependent lipogenesis: beneficial effect on hepatic steatosis in a high fat-high sucrose diet NAFLD obese rat model
}

Laura Braud, Sylvain Battault, Grégory Meyer, Alessandro Nascimento, Sandrine Gaillard, Georges De Sousa, Roger Rahmani, Catherine Riva, Martine Armand, Jean-Michel Maixent, et al.

\section{To cite this version:}

Laura Braud, Sylvain Battault, Grégory Meyer, Alessandro Nascimento, Sandrine Gaillard, et al.. Antioxidant properties of tea blunt ROS-dependent lipogenesis: beneficial effect on hepatic steatosis in a high fat-high sucrose diet NAFLD obese rat model. Journal of Nutritional Biochemistry, 2016, 40, pp.95-104. 10.1016/j.jnutbio.2016.10.012 . hal-01425507

\section{HAL Id: hal-01425507 \\ https://hal-amu.archives-ouvertes.fr/hal-01425507}

Submitted on 10 Jan 2022

HAL is a multi-disciplinary open access archive for the deposit and dissemination of scientific research documents, whether they are published or not. The documents may come from teaching and research institutions in France or abroad, or from public or private research centers.
L'archive ouverte pluridisciplinaire HAL, est destinée au dépôt et à la diffusion de documents scientifiques de niveau recherche, publiés ou non, émanant des établissements d'enseignement et de recherche français ou étrangers, des laboratoires publics ou privés.

\section{(1) (1) $\$$}

Distributed under a Creative Commons Attribution - NonCommercial - NoDerivatives 44.0 


\section{Accepted Manuscript}

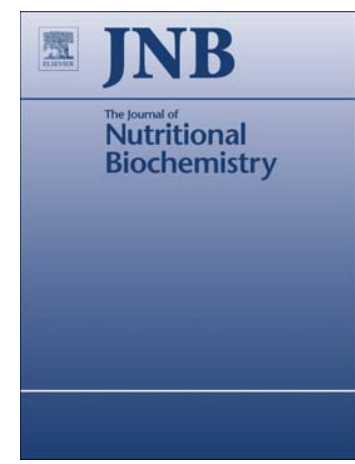

Antioxidant properties of tea blunt ROS-dependent lipogenesis: beneficial effect on hepatic steatosis in a high fat-high sucrose diet NAFLD obese rat model

Laura Braud, Sylvain Battault, Grégory Meyer, Alessandro Nascimento, Sandrine Gaillard, Georges de Sousa, Roger Rahmani, Catherine Riva, Martine Armand, Jean-Michel Maixent, Cyril Reboul

PII:

S0955-2863(16)30643-X

DOI: doi: 10.1016/j.jnutbio.2016.10.012

Reference: JNB 7671

To appear in: The Journal of Nutritional Biochemistry

Received date: 28 April 2016

Revised date: 19 September 2016

Accepted date:

5 October 2016

Please cite this article as: Braud Laura, Battault Sylvain, Meyer Grégory, Nascimento Alessandro, Gaillard Sandrine, de Sousa Georges, Rahmani Roger, Riva Catherine, Armand Martine, Maixent Jean-Michel, Reboul Cyril, Antioxidant properties of tea blunt ROS-dependent lipogenesis: beneficial effect on hepatic steatosis in a high fat-high sucrose diet NAFLD obese rat model, The Journal of Nutritional Biochemistry (2016), doi: 10.1016/j.jnutbio.2016.10.012

This is a PDF file of an unedited manuscript that has been accepted for publication. As a service to our customers we are providing this early version of the manuscript. The manuscript will undergo copyediting, typesetting, and review of the resulting proof before it is published in its final form. Please note that during the production process errors may be discovered which could affect the content, and all legal disclaimers that apply to the journal pertain. 


\section{Antioxidant properties of tea blunt ROS-dependent lipogenesis: beneficial effect on hepatic steatosis in a high fat-high sucrose diet NAFLD obese rat model}

Laura Braud ${ }^{\mathrm{a}^{*}}$, Sylvain Battault ${ }^{\mathrm{b}}$, Grégory Meyer ${ }^{\mathrm{b}}$, Alessandro Nascimento ${ }^{\mathrm{b}}$, Sandrine Gaillard $^{\mathrm{c}}$, Georges de Sousa ${ }^{\mathrm{d}}$, Roger Rahmani ${ }^{\mathrm{d}}$, Catherine Riva ${ }^{\mathrm{b}}$, Martine Armand ${ }^{\mathrm{e}}$, JeanMichel Maixent ${ }^{\mathrm{a} £}$, Cyril Reboul ${ }^{\mathrm{b} £}$

aaboratoire Protee, Equipe EB2M, Université de Toulon, avenue de l'Université, 83130 La Garde, France

${ }^{\mathrm{b}}$ Laboratoire de Pharm-Ecologie Cardiovasculaire (EA4278), Faculty of Sciences, Avignon University, 33 rue Louis Pasteur, 84000 Avignon, France

'Plateforme BioTechServices, Université de Toulon, avenue de l'Université, 83130 La Garde, France

dUMR 1331 TOXALIM (Research Centre in Food Toxicology), Institut National de la Recherche Agronomique (INRA), Laboratory of Xenobiotic's Cellular and Molecular Toxicology, 400 route des Chappes, 06903 Sophia-Antipolis, France

eAix-Marseille Université, CNRS, CRMBM UMR 7339, F-13385 Marseille, France

${ }^{\mathfrak{f}}$ Senior co-authors

*Corresponding author: Laura Braud, Laboratoire PROTEE, EB2M, Université de Toulon, avenue de l'Université, 83130 La Garde, France. Phone: +33 04941426 70. Fax: +33 0494 142045

Email address: lbraud01 @ gmail.com (‥ Braud)

Running title: Anti-lipogenic action of tea antioxidant molecules 


\begin{abstract}
Oxidative stress could trigger lipid accumulation in liver and thus hepatic steatosis. Tea is able to prevent liver disorders but a direct link between antioxidant capacities and prevention of steatosis has not been reported yet. We aimed to investigate such relationship in a rat model of high fat-high sucrose diet (HFS)-induced obesity and to explore more deeply the mechanisms in isolated hepatocytes. Wistar rats were divided into a Control group (standard diet), a HFS group (high fat-sucrose diet) and a HFS+Tea group (HFS diet with ad-libitum access to tea drink). Body weight, fat mass, glycemic parameters in blood, lipid and oxidative stress parameters in blood and liver were measured in each group after 14 weeks. Isolated hepatocytes were treated with the reactive oxygen species (ROS) inducer $t$-BHP in the presence or not of antioxidants (tempol or tea), and superoxide anion production and lipid accumulation were measured using specific fluorescent probes. We reported that the HFS diet highly increased hepatic lipids content, while tea consumption attenuated steatosis and improved the oxidative status (decrease in hepatic oxidative stress, increase in plasma total antioxidant capacity). The role of antioxidant properties of tea in such phenomenon was confirmed in primary cultured rat hepatocytes. Indeed, the increase of mitochondrial ROS production with $t$-BHP resulted in lipid accumulation in hepatocytes (positive linear regression), and antioxidants (tempol or tea) normalized both. We reported that the antioxidant properties of tea protect rats from an obesogenic HFS diet-induced hepatic steatosis by counteracting the ROS-dependent lipogenesis.
\end{abstract}

\title{
Keywords
}

NAFLD; Camellia sinensis; Anti-lipogenesis; Antioxidant; mitochondrial ROS; obesogenic diet 


\section{Introduction}

Non-alcoholic fatty liver disease (NAFLD) refers to a spectrum of chronic liver diseases ranging from hepatic steatosis to non-alcoholic steatohepatitis progressing to fibrosis and ultimately to cirrhosis [1]. Since NAFLD has been strongly associated with obesity, insulin resistance, hypertension and dyslipidemia, and is now considered as the liver metabolic syndrome manifestation, it represents nowadays the most common and emerging chronic liver disease [2]. Because of the difficulty of the diagnosis, the exact global incidence of NAFLD is unknown. However, it has been estimated in Western countries that the prevalence of NAFLD is about $30 \%$ while it is about $5-18 \%$ in Asia [3]. In obese subjects, NAFLD is characterized by the accumulation of lipids in the liver which is considered as the "first hit" in the progression from steatosis to steatohepatitis. The "second hit" is dependent on increased oxidative stress and characterized by lipid peroxidation, inflammation, mitochondrial dysfunction and hepatic injury [4]. Unfortunately, today, there are only limited options for the treatment of NAFLD, including lifestyle modification, weight management, vitamin $\mathrm{E}$ and thioglitazone treatment $[5,6]$.

Epidemiological and clinical studies have shown that tea consumption was associated with decrease liver disorders $[7,8]$. But the underlying mechanisms associated with the hepatoprotective effects of tea are still not clear. Indeed, tea is recognized for its antioxidant properties, mainly due to tea polyphenols, which contribute to protect ob/ob mice from hepatic steatosis [9]. The beneficial effects of tea are also reported to be due to direct antilipogenesis properties $[10,11]$. However, despite the described role of oxidative stress in hepatic lipogenesis [12-14], the contribution of the antioxidant properties of tea to its antilipogenesis action has never been investigated.

The aim of this work was to test whether the antioxidant properties of tea could be in part responsible of its anti-lipogenesis effects. For this purpose, we used an obesogenic high fathigh sucrose diet in rats to examine the effect of tea on hepatic lipid parameters, antioxidant capacity and lipid peroxidation, together with a primary culture of rat hepatocytes to deeply explore the underlying mechanisms. 


\section{Materials and methods}

\section{Animals and experimental design}

Seven-week-old male Wistar rats weighing about $200 \mathrm{~g}$ ( $\pm 25 \mathrm{~g})$ were obtained from Janvier (France). Three rats were housed per cage under controlled conditions of temperature $\left(21^{\circ} \mathrm{C} \pm\right.$ $1)$, hygrometry $(60 \% \pm 10)$ and lighting (12 hours a day). Animals were acclimatized to the animal laboratory for seven days before the start of the experiment. Rats were randomly divided into three groups: Control group, high fat-high sucrose diet (HFS) group and HFS+Tea group ( $n=15$ per group). The control group was fed with a standard diet while the others consumed high fat diet (60\% fat Purified Diet $230 \mathrm{HF}$ ). Standard diet (A04) and high fat diet (Purified Diet $230 \mathrm{HF}$ ) were purchased from SAFE Diet (Augy, France). Sucrose was purchased from Sigma Chemical Co. (St Louis, MO, USA) and supplied at the dose of $10 \%$ in the drink for the HFS groups. Tea infusion was supplied as drink for the HFS+Tea group. Rats were provided free access to food and fluid for 14 weeks and dietary consumptions were recorded three times each week. Consumption of tea was about $50 \mathrm{~mL}$ per day per rat. The composition of experimental diets is shown in Table 1. All along the protocol rats were weighed weekly. Rats were sacrificed at the end of 14 weeks and after a $12 \mathrm{~h}$ fast. Blood was collected for biochemistry analysis. Total dorsal and epididymal fat were removed and weighed as index of visceral obesity. Livers were removed in order to perform biochemical measurements. All animals received care according to institutional guidelines, and all experiments were approved by the Institutional Ethics committee (Agreement number A-84007-2).

\section{Tea infusion characterization and preparation}

Hao Ling ${ }^{\circledR}$ tea is a blend of green, oolong and pu-erh tea leaves coming from Zhejiang, Fujian and Yunnan province (China), respectively. This blend of teas is elaborated following a confidential manufacturing process by the french company "Thés de la Pagode" (Paris). The infusions were prepared by pouring $200 \mathrm{~mL}$ of distilled water at $100{ }^{\circ} \mathrm{C}$ on $20 \mathrm{~g}$ of tea leaves mixture powder and brewing for $15 \mathrm{~min}$. The tea infusion obtained was then diluted in 4 volumes of tap water to obtain a tea infusion at human nutritional doses. For culture cell experiments, the infusion was lyophilized using HetoPowerDry LL1500 (Thermo Electron, USA), and the freeze-dried aqueous tea extracts was stored at $-20^{\circ} \mathrm{C}$ until analysis. Total phenolic content and quantification of aqueous tea extracts compounds were performed as 
previously described [15]. In the diluted aqueous tea infusion, total polyphenols, catechins, gallic acid and caffeine content were $126.6 \mathrm{mg}, 10.7 \mathrm{mg}, 8.4 \mathrm{mg}$ and $41.12 \mathrm{mg}$ per $100 \mathrm{~mL}$ (Table 2), respectively. The amount of the main catechins, epigallocatechin, epicatechin, epigallocatechin gallate and epicatechin gallate were $1.2 \mathrm{mg}, 2.2 \mathrm{mg}, 5.5 \mathrm{mg}$ and $1.8 \mathrm{mg}$ per $100 \mathrm{~mL}$ of aqueous tea infusion respectively (Table 2).

\section{Fasting blood glucose, glucose and insulin tolerance tests.}

An Oral Glucose Tolerance Test (OGTT) was performed at the end of the $13^{\text {th }}$ week of the chronic protocol. First, blood was obtained via tail clip to assess fasting blood glucose (Caresens $^{\circledR} \mathrm{N}$, DinnoSanteTM). Then, rats received a glucose solution $(2 \mathrm{~g} / \mathrm{kg})$ by oral gavage, and blood glucose was measured at 20, 40, 60, 80, 100 and $120 \mathrm{~min}$ after the glucose oral gavage. On the following week, intra-peritoneal Insulin Tolerance Test (ITT) was performed. First, fasting blood glucose was measured and then rats received an intraperitoneal injection of insulin solution $(2 \mathrm{UI} / \mathrm{kg})$ and blood glucose was measured at 30, $60,90,120,150,180$ and $210 \mathrm{~min}$ after insulin injection.

\section{Analysis of plasma lipids and ALT concentrations}

Enzymatic assays (kits from Roche, Indianapolis, USA) were performed on Cobas 6000 analyser (Roche) for measuring triglycerides (TG), low-density lipoprotein (LDL), highdensity lipoprotein (HDL) and alanine transaminase (ALT). Non-esterified fatty acid (NEFA) was performed with Randox kit (Randox Laboratories, UK).

\section{Measurement of liver lipid content}

Portions of liver samples from each group of rats were weighed and homogenized in phosphate buffered saline (PBS) (0.01 M phosphate, 0.0027 M potassium chloride, $0.137 \mathrm{M}$ sodium chloride at $\mathrm{pH}$ 7) using a IKA T10 basic ULTRA TURRAX Homogenizer (SigmaAldrich). Lipid extraction from homogenates was performed using a mixture of chloroform/methanol 2/1 v,v [16], and contents of triglycerides and of the total cholesterol (PAP 150 kit and RTU kit, respectively, from Biomérieux, Lyon, France), and of NEFA (Randox kit, Randox Laboratories, United-Kingdom) were quantified.

\section{Analysis of mRNA expression}

After an initial extraction step by mixing Extract-All (Eurobio, France) and chloroform to samples, hepatocytes total RNA purification was performed with a column extraction Kit 
(RNeasy Mini ${ }^{\circledR}$, Qiagen, Germany). A supplementary rDNase treatment was added to the manufacturer's instructions in order to remove any genomic DNA contamination. This one was excluded by a control PCR amplification of RNA. Double-strand cDNA was synthesized from total RNA according to the manufacturer's manual (Omniscript ${ }^{\circledR}$, Qiagen, Germany). SYBR Green real-time PCR quantifications were performed using commercially available $\mathrm{RT}^{2}$ Profiler ${ }^{\mathrm{TM}}$ PCR Arrays from Qiagen (Germany) containing primer pairs that have been experimentally validated to ensure gene-specificity and high amplification efficiency on a LightCycler 480 (Roche, Germany). Levels of mRNA, expressed as relative mRNA levels compared with control diet-fed rats, were calculated after normalization to RPL17 reference gene.

\section{Hepatic lipid peroxidation, and antioxidant capacity}

The hepatic level of lipid peroxidation expressed as hepatic malondialdehyde (MDA) equivalent was evaluated using QuantiChrom Thiobarbituric acid reactive substances (TBARS) and the Glutathione Peroxidase (GPx) activity was measured with GPx assay kits from BioAssay Systems (USA). Total hepatic antioxidant capacity was assessed using Antioxidant Assay Kit from Sigma Chemical Co. (St Louis, MO, USA). Total antioxidant capacity of plasma was determined using OxiSelect ${ }^{\mathrm{TM}}$ TAC assay kit from Cell BioLabs (USA).

\section{Measurement of lipid accumulation and mitochondrial superoxide anion on primary culture of rat hepatocytes}

Hepatocytes were freshly isolated from rattus norvegicus ofa male rats weighing 180 to $200 \mathrm{~g}$ (Iffa Credo, France) by collagenase perfusion of the liver [17]. The national guidelines for care and use of research animals were followed (agreement number A 13823, French Ministry of Agriculture). Hepatocytes were seeded as previously described [15]. Cells were then treated for $12 \mathrm{~h}$ with $100 \mu \mathrm{L}$ of various concentrations of aqueous tea extract $(100,250$ and $500 \mu \mathrm{g} / \mathrm{mL}$ ) with or without $120 \mu \mathrm{M}$ of exogenous oxidative stress inducer $t$-BHP (tertbutyl hydroperoxide in DMSO solution, both purchased from Sigma-Aldrich, France). Such doses were selected after testing several concentrations (from $100 \mu \mathrm{M}$ to $1 \mathrm{mM}$ ) in order to generate sufficient oxidative stress without killing all cells (data not shown). The relative hepatocyte lipid accumulation and mitochondrial superoxide anion were measured using Neutral Lipid Stains (LipidTox) green staining and Mitochondrial superoxide indicator (MitoSox) red staining probes (Life Technologies, France), respectively as previously 
described [15]. Control i.e. "non-treated" cells consisted of medium, water or DMSO depending on the experiment. DMSO did not interfere with the experiment when present (data not shown).

\section{Statistical analysis}

Data are expressed as mean values \pm standard error of the mean (SEM). Statistical analysis was performed by one-way analysis of variance (ANOVA) with Fisher multiple comparison test. The result were considered significant if the p-value was $<0.05$. 


\section{Results}

\section{Effects of tea on HFS diet-induced obesity}

Seven-week-old male Wistar rats fed a high fat-sucrose diet for 14 weeks gained significantly more weight $(+143 \%)$ than rats fed with a standard diet $(+117 \%)$, and this increase in weight was similar in the HFS+Tea group (Figure 1A). The HFS diet increased the epididymal and the dorsal fat mass when compared to Ctrl rats, and tea consumption had no preventive effect (Figure 1A). Moreover, fasting blood glucose as well as glycemic response to an oral glucose tolerance test (OGTT) (Figure 1B) and an intraperitoneal insulin tolerance test (Supplemental Figure 1) were higher in HFS compared to Ctrl rats. Tea consumption had no effect on the regulation of those metabolic parameters (Figure 1B). Regarding dyslipidemia, a classic feature of obesity, the HFS diet resulted in a significant increase in plasma non esterified fatty acid (NEFA), triglycerides (TG) and LDL-cholesterol levels compared to control diet $(+50,+48,+40 \%$, respectively) with no significant effect of tea supplementation (Figure 1C). In addition, plasma HDL-cholesterol level was significantly decreased in HFS group (-16\%) compared to Ctrl group leading to a significant 1.5 fold increase in LDL/HDL ratio (Figure 1C). Tea consumption normalized plasma levels of HDLcholesterol caused by the HFS diet and consequently normalized the LDL/HDL ratio to Ctrl values. Altogether, these results showed that in our reliable rat model of HFS-diet induced metabolic syndrome, tea consumption exerts only beneficial effects on specific blood lipid profile (HDL and LDL/HDL).

\section{Tea attenuates obesogenic HFS diet-induced hepatic steatosis}

The HFS diet induced a significant increase in liver weight (1.15 fold) and in lipid content as shown by its color change and the increase of lipid droplets with oil red $\mathrm{O}$ staining (Figure $2 \mathrm{~A}$ and $2 \mathrm{~B})$. In agreement with this result, higher liver levels of total lipid (+ 2.3 fold), triglycerides $(+3.5$ fold $)$, total cholesterol $(+7$ fold $)$ and non-esterified fatty acid (NEFA) (+ 1.8 fold) were measured in HFS compared to Ctrl rats (Figure 2B-F). Tea consumption led to a significant decrease in several of those parameters, NEFA (- 24.6\%), TG (- $24.3 \%)$ and total cholesterol (- 43.6\%) (Figure 2D-F), while a trend for reduction was observed for the liver's weight and total lipid content (Figure 2B-C), as well as for alanine transaminase (ALT) plasmatic levels, a reliable index of liver injury (Figure 2G). These results clearly 
highlight that tea consumption alleviates hepatic lipid accumulation and thus injury induced by the HFS diet.

\section{Tea attenuates obesogenic HFS diet-induced hepatic oxidative stress}

To explore the mechanisms by which tea decrease hepatic lipogenesis in vivo and the potential role of oxidative stress in such effects, we next evaluated whether tea treatment modulates the oxidative status in HFS liver. Indeed, hepatic steatosis has been described to be associated with increased hepatic oxidative stress and lipid peroxidation [1], however, whether the beneficial effects of tea on hepatic steatosis [18] are related to its antioxidant properties has never been challenged. First, we reported that lipid peroxidation marker MDA, an accurate index of oxidative stress, was increased in HFS rat livers compared to the controls (Figure 3A). This was associated with reduced hepatic total antioxidant capacity in HFS compared to Ctrl (Figure 3B) without change in plasma total antioxidant capacity (Figure 3C). Interestingly, in tea treated HFS rats, MDA levels in liver were significantly reduced compared to untreated HFS rats (Figure 3A). However, hepatic total antioxidant capacity was not increased by tea consumption (Figure 3B), while plasma antioxidant capacity was increased in HFS+Tea group compared to control and HFS rats (Figure 3C). Furthermore, hepatic MDA was strongly positively correlated with hepatic total lipid content $\left(\mathrm{R}^{2}=0.80, p<0.0001\right)$ (Figure 3D). Altogether, those results strongly suggest that tea limit increased liver oxidative stress in HFS rats, which correlates with reduced total lipid content.

\section{Tea attenuates lipogenesis in primary culture of rat hepatocytes through its antioxidant properties}

Despite we reported a strong relationship between liver oxidative stress and lipid content, whether increased ROS production contributes to increased lipid content in hepatocytes remains not clear. Thus, to evaluate whether tea acts by modulating a ROS-dependent lipid accumulation in hepatocytes, we next evaluated in primary culture of rat hepatocytes the effect of the $t$-BHP oxidative stress inducer on lipid accumulation in presence or not of tea or tempol. The exposure to $t$-BHP is well known to simulate a situation of increased oxidative stress by stimulating mitochondrial ROS overproduction within the cell [19]. Consistently, twelve hours incubation with $t$-BHP induced a 2.5 fold increase in $\mathrm{O}_{2}^{-{ }^{-}}$production as demonstrated by MitoSox fluorescence (Figure 4A). Interestingly, this was associated with a 1.47 fold increase in lipid accumulation as demonstrated by LipidTox fluorescence (Figure 4B). To test whether such increased production of $\mathrm{O}_{2}{ }^{--}$could contribute to lipid accumulation 
within cells, we next used the well-known antioxidant (superoxide dismutase mimetic) tempol (4-hydroxy-2,2,6,6-tetramethylpiperidine-1-oxyl) [20] to counteract oxidative stress induced by $t$-BHP. We observed that tempol completely prevented the increased in $\mathrm{O}_{2}{ }^{-}$ production induced by $t$-BHP treatment (Figure 4A) and subsequently decreased lipid accumulation within hepatocytes (Figure 4B). These results suggest that the increased in ROS production in hepatocytes strongly contributes to the increase in lipid accumulation. Thus, considering the beneficial effect of tea consumption on both oxidative stress and lipid accumulation in the liver, we next evaluated whether incubation of primary culture of rat hepatocytes with tea could also prevent lipid accumulation during $t$-BHP-induced $\mathrm{O}_{2}{ }^{-}$ production. The addition of the lowest dose $(100 \mu \mathrm{g} / \mathrm{mL})$ of aqueous tea extract partially decreased $\mathrm{O}_{2}{ }^{--}$production induced by $t$-BHP treatment while highest doses (250 and 500 $\mu \mathrm{g} / \mathrm{mL}$ ) completely prevented this phenomenon in a dose-dependent manner (Figure 4A). In line with the results obtained with tempol and with reduced $\mathrm{O}_{2}^{--}$production in hepatocytes with tea extract, the highest doses of tea extract ( 250 and $500 \mu \mathrm{g} / \mathrm{mL}$ ) fully decreased the lipid accumulation induced by $t$-BHP (Figure 4B). Finally, we confirmed that MitoSox Red fluorescence was positively correlated with LipidTox fluorescence in hepatocytes $\left(\mathrm{R}^{2}=0.58\right.$, $p<0.0001$ ) (Figure 4C). Altogether, these results demonstrated that the decreased in $\mathrm{O}_{2}{ }^{-}$ production using antioxidant agents prevented lipid accumulation in isolated hepatocytes and that the hepatic anti-lipogenic properties of tea could be mediated through its antioxidant properties.

\section{Discussion}

The present study aimed to evaluate whether the beneficial effects of tea on obesity induced NAFLD could be related to its antioxidant properties. We report here that at the cellular level, tea is able to counteract a ROS-dependent lipid accumulation in a primary culture of rat hepatocytes and, in-vivo, that tea consumption was able to reduce liver oxidative stress and to limit the development of hepatic steatosis in a diet-induced obese rat model.

NAFLD refers to a spectrum of chronic liver diseases ranging from simple hepatic steatosis to non-alcoholic steatohepatitis progressing to fibrosis and ultimately to cirrhosis [1]. Two decades ago, the so-called "two hits" theory was presented by Christopher Day and Oliver James to describe the development of NAFLD [4]. This model suggested that hepatic 
steatosis is a prerequisite for subsequent events that lead to oxidative stress, lipid peroxidation and finally liver injury. Although many studies have reported results corroborating this theory [21,22], the pathogenesis of the disease is still poorly understood. Furthermore, several recent reviews nuance the involvement of hepatic steatosis in the initiation of the pathogenesis and suggest a causal role of oxidative stress [23-25]. Especially, mitochondrial dysfunction with subsequent redox alterations in the early phases of NAFLD has been observed in rats [26]. This also constitutes a main feature of obesity and insulin-resistance [27,28], which could thus trigger NAFLD in obese subjects. Mitochondrial dysfunction is recognized to be strongly associated with increased intracellular ROS production due to an impairment of mitochondrial respiratory chain $[29,30]$, which could thus play a key role in redox-dependent modulation of lipid metabolism that could precede the development of hepatic steatosis [24]. In line with this hypothesis, we reported here that the only use of pro-oxidant $t$-BHP, well-known to alter mitochondrial function in hepatocytes [15], induced a significant increase in mitochondrial $\mathrm{O}_{2}{ }^{-}$which correlates with increased lipid accumulation in a primary culture of rat hepatocytes. Moreover, we also reported that as previously published in a rat model of NAFLD [31], liver steatosis was associated with increased oxidative stress together with an increase in total lipid content. Thus, oxidative stress by itself may constitute a key trigger of hepatic lipid accumulation in the liver.

The accumulation of hepatic lipid content have been shown to be mediated by the increase of key lipogenesis gene expression such as PPar $\gamma$, Clebpa and Srebplc this latter inducing the expression of Fas and Acc, two rate-limiting enzymes of lipogenesis [32-34]. This is also evident from our work (Supplemental Figure 2A), and interestingly, some of those genes seem sensitive to redox modulation. Indeed, depletion of superoxide dismutase (SOD), the antioxidant enzyme which catalyses the transformation of $\mathrm{O}_{2}{ }^{-}$into hydrogen peroxide $\left(\mathrm{H}_{2} \mathrm{O}_{2}\right)$, induced lipid accumulation in primary culture of mouse hepatocytes [13] and in mouse liver by enhancing Srebplc protein synthesis [14]. Same results were obtained in HepG2 cells line in which oxidative stress was induced by $\mathrm{H}_{2} \mathrm{O}_{2}$ [35]. All these data highly suggest that ROS over-production is implicated in the increase of lipogenesis gene expression.

Considering a plausible direct role of oxidative stress on lipogenesis genes and lipid accumulation in hepatocytes, we postulated that antioxidant treatment per itself could have beneficial effect on hepatic lipid accumulation. Tea is the second most consumed beverage worldwide, after water, and is obtained from brewing leaves of Camellia sinensis. It is a good 
source of powerful antioxidant molecules, especially phenolic compounds such as catechins and phenolic acids $[36,37]$. In line with numerous studies on the antioxidant effects of tea [9,38-41], in our model, the level of total polyphenols $(126 \mathrm{mg} / 100 \mathrm{~mL})$ could strongly explain its effects on ROS production. Indeed, tea polyphenols have been demonstrated to attenuate oxidative stress by acting as free-radical scavengers and modulating pro-oxidant and antioxidant cellular enzymes [9,39]. In particular, it has been described to attenuate oxidative stress in NAFLD rats $[9,40,41]$. In addition, others reported that tea attenuated hepatic steatosis in NAFLD rats due to its anti-lipogenesis properties i.e. by decreasing the expression of genes involved in lipogenesis [10,11]. However, the link between antioxidant properties of tea and its lipid-lowering effects has never been demonstrated. In our study, we provided evidence on cultured hepatocytes that the tea used in the present work has good antioxidant properties, since $\mathrm{O}_{2}{ }^{-}$production, resulting from the ROS inducer $t$-BHP, was normalized to the same extent with tempol and tea. This is in line with prior results from our team showing that on cultured hepatocytes the same tea extract protect mitochondrial functional state during oxidative stress [15]. In our work, those antioxidant properties of tea are also involved in the modulation of lipid metabolism. Indeed, tea significantly decreased the lipid accumulation in liver and in isolated hepatocytes, which was positively correlated with decreased oxidative stress both in vivo and in vitro. Some studies have reported that tea could enhance hepatic enzymatic antioxidant defenses such as glutathione peroxidase, superoxide dismutase or catalase and therefore participate to its antioxidant properties $[9,42]$. However, in our study, hepatic total antioxidant capacity and hepatic glutathione peroxidase activity (data not shown) were not improved by tea, while the plasma total antioxidant capacity was increased suggesting that antioxidant activity of tea could intervene upon the systemic circulation to protect the liver from oxidative stress. Indeed, systemic markers of lipid peroxidation and antioxidants in patients with NAFLD have been reported to be negatively correlated [43].

The beneficial effects of tea are also discussed in the literature as being mediated by some effects on obesity and insulin-sensitivity in adipose tissue [44,45], which could explain its effects on hepatic steatosis. In contrast, in our study, tea consumption did not improve HFS diet-induced weight gain or insulin-resistance (Supplemental Figure 1) and thus did not explain its effect on hepatic steatosis by this way. Moreover, no benefit in plasma triglyceride and non-esterified fatty acid levels was observed. On the other hand, we found that our tea ameliorated hypercholesterolemia by normalizing plasma HDL levels. However, whether this 
is a consequence of the beneficial effects of tea or at least a contributor to the observed reduction in hepatic steatosis, and consequently improvement in liver functioning, remains to be clarified. Nevertheless, a lipotropic effect of polyphenols especially catechins have been reported and we can envisage that our antioxidant molecules acted also by favoring the efflux of lipids from the liver, in order to help diminishing fat accumulation, explaining the unchange in the high level of plasma triglycerides, NEFA and cholesterol (via the secretion of VLDL), and the increase in HDL [46]. Further studies will be needed to improve the understanding of the beneficial effects of tea on LDL/HDL ratio which constitute a key index of cardiovascular risk factors [47]. Finally, hepatic steatosis could also be explained by reduced $\beta$-oxidation of fatty acids [1]. Accordingly, mRNA level of Acox, the first enzyme implicated in the fatty acid $\beta$-oxidation, was lower in steatotic livers (Supplemental Figure 2B). However, since our tea supplementation had no effect on Acox gene expression, the impact of an improved $\beta$-oxidation of fatty acids explaining the decrease in fat accumulation in our study is doubtful (Supplemental Figure 2B). Altogether, our findings reveal that tea attenuates hepatic steatosis mainly by decreasing hepatic lipogenesis via independent mechanisms of obesity, insulin resistance and fatty acid $\beta$-oxidation. Those elements further support our hypothesis of a direct key role of tea antioxidant properties in its anti-lipogenesis action.

\section{Study limitation}

In our model of HFS diet, mild obesity was clearly associated with classical features of NAFLD. However, as evidenced by the lack of increase in ALT, used as an index of hepatocellular injuries [6,48], our HFS diet was not associated with severe hepatic disorders. However, it is of interest that the ALT levels may be normal in patients with steatohepatitis or cirrhosis [5,49]. NAFLD can presents cytonecrotic profile but also a cholestasis profile with increase in the gamma-glutamyltranspeptidase (GGT) values. In our, study, GGT were not measured. However, ALT and GGT enzyme levels are positively correlated during NAFLD development [50]. We can thus stipulate that in our model, NAFLD was not associated with cholestasis, but it remains to be explored. Thus, in our model, tea aqueous extract was efficient to prevent the development of an early stage of NAFLD. Whether this strategy would remain able to protect the illness in its advanced stage needs further experiments. 


\section{Conclusion}

To conclude, our experiments demonstrated that an obesogenic diet contributes to increase oxidative stress in the liver with subsequent effect on lipid accumulation and the development of steatosis. We also reported that at the cellular level, antioxidant treatment contributes to prevent the ROS-dependent increase in lipid accumulation. Finally, we reported for the first time that tea exerts its beneficial effect on the development of NAFLD in part directly through its antioxidant properties (Figure 5). Consequently, these findings demonstrate that tea has potentially beneficial effects on the prevention of NAFLD in rats, and more specifically that tea antioxidant molecules could be plausibly considered as therapeutic strategies to prevent the hepatic steatosis during the development of NAFLD in obese subjects. 


\section{Disclosures}

This study was partly supported by Thés de la Pagode Company.

\section{Acknowledgements}

The authors wish to thank Jean-Yves Tano for his help in evaluating the manuscript for English.

\section{References}

[1] Willebrords J, Pereira IVA, Maes M, Crespo Yanguas S, Colle I, Van Den Bossche B, et al. Strategies, models and biomarkers in experimental non-alcoholic fatty liver disease research. Prog Lipid Res 2015;59:106-25. doi:10.1016/j.plipres.2015.05.002.

[2] Vernon G, Baranova A, Younossi ZM. Systematic review: the epidemiology and natural history of non-alcoholic fatty liver disease and non-alcoholic steatohepatitis in adults. Aliment Pharmacol Ther 2011;34:274-85. doi:10.1111/j.1365-2036.2011.04724.x.

[3] Masarone M, Federico A, Abenavoli L, Loguercio C, Persico M. Non Alcoholic Fatty Liver: Epidemiology and Natural History. Rev Recent Clin Trials 2014;9:126-33.

[4] Day CP, James OFW. Steatohepatitis: A tale of two "hits"? Gastroenterology 1998;114:842-5. doi:10.1016/S0016-5085(98)70599-2.

[5] Ahmed M. Non-alcoholic fatty liver disease in 2015. World J Hepatol 2015;7:1450-9. doi:10.4254/wjh.v7.i11.1450.

[6] EASL-EASD-EASO Clinical Practice Guidelines for the management of non-alcoholic fatty liver disease. J Hepatol 2016;64:1388-402. doi:10.1016/j.jhep.2015.11.004.

[7] Kuriyama S. The relation between green tea consumption and cardiovascular disease as evidenced by epidemiological studies. J Nutr 2008;138:1548S-1553S.

[8] Nakachi K, Matsuyama S, Miyake S, Suganuma M, Imai K. Preventive effects of drinking green tea on cancer and cardiovascular disease: epidemiological evidence for multiple targeting prevention. BioFactors Oxf Engl 2000;13:49-54.

[9] Park HJ, DiNatale DA, Chung M-Y, Park Y-K, Lee J-Y, Koo SI, et al. Green tea extract attenuates hepatic steatosis by decreasing adipose lipogenesis and enhancing hepatic antioxidant defenses in ob/ob mice. J Nutr Biochem 2011;22:393-400. doi:10.1016/j.jnutbio.2010.03.009.

[10] Axling U, Olsson C, Xu J, Fernandez C, Larsson S, Ström K, et al. Green tea powder and Lactobacillus plantarum affect gut microbiota, lipid metabolism and inflammation in high-fat fed C57BL/6J mice. Nutr Metab 2012;9:105. doi:10.1186/1743-7075-9-105.

[11] Lin C-L, Huang H-C, Lin J-K. Theaflavins attenuate hepatic lipid accumulation through activating AMPK in human HepG2 cells. J Lipid Res 2007;48:2334-43. doi:10.1194/jlr.M700128-JLR200.

[12] Hassan W, Rongyin G, Daoud A, Ding L, Wang L, Liu J, et al. Reduced Oxidative Stress Contributes to the Lipid Lowering Effects of Isoquercitrin in Free Fatty Acids Induced Hepatocytes. Oxid Med Cell Longev 2014;2014:e313602. doi:10.1155/2014/313602.

[13] Lee J, Homma T, Kurahashi T, Kang ES, Fujii J. Oxidative stress triggers lipid droplet accumulation in primary cultured hepatocytes by activating fatty acid synthesis. Biochem Biophys Res Commun 2015. doi:10.1016/j.bbrc.2015.06.121. 
[14] Wang L, Jiang Z, Lei XG. Knockout of SOD1 altered murine hepatic glycolysis, gluconeogenesis, and lipogenesis. Free Radic Biol Med 2012;53:1689-96. doi:10.1016/j.freeradbiomed.2012.08.570.

[15] Braud L, Peyre L, de Sousa G, Armand M, Rahmani R, Maixent J-M. Effect of Brewing Duration on the Antioxidant and Hepatoprotective Abilities of Tea Phenolic and Alkaloid Compounds in a t-BHP Oxidative Stress-Induced Rat Hepatocyte Model. Mol Basel Switz 2015;20:14985-5002. doi:10.3390/molecules200814985.

[16] Folch J, Lees M, Sloane Stanley GH. A simple method for the isolation and purification of total lipides from animal tissues. J Biol Chem 1957;226:497-509.

[17] de Sousa G, Dou M, Barbe D, Lacarelle B, Placidi M, Rahmani R. Freshly isolated or cryopreserved human hepatocytes in primary culture: Influence of drug metabolism on hepatotoxicity. Toxicol In Vitro 1991;5:483-6. doi:10.1016/0887-2333(91)90077-Q.

[18] Masterjohn C, Bruno RS. Therapeutic potential of green tea in nonalcoholic fatty liver disease. Nutr Rev 2012;70:41-56. doi:10.1111/j.1753-4887.2011.00440.x.

[19] Kučera O, Endlicher R, Roušar T, Lotková H, Garnol T, Drahota Z, et al. The Effect of tert-Butyl Hydroperoxide-Induced Oxidative Stress on Lean and Steatotic Rat Hepatocytes In Vitro. Oxid Med Cell Longev 2014;2014. doi:10.1155/2014/752506.

[20] Wilcox CS. Effects of tempol and redox-cycling nitroxides in models of oxidative stress. Pharmacol Ther 2010;126:119-45. doi:10.1016/j.pharmthera.2010.01.003.

[21] Angulo P. Nonalcoholic Fatty Liver Disease. N Engl J Med 2002;346:1221-31. doi:10.1056/NEJMra011775.

[22] Browning JD, Horton JD. Molecular mediators of hepatic steatosis and liver injury. J Clin Invest 2004;114:147-52. doi:10.1172/JCI200422422.

[23] Polimeni L, Del Ben M, Baratta F, Perri L, Albanese F, Pastori D, et al. Oxidative stress: New insights on the association of non-alcoholic fatty liver disease and atherosclerosis. World J Hepatol 2015;7:1325-36. doi:10.4254/wjh.v7.i10.1325.

[24] Serviddio G, Bellanti F, Vendemiale G. Free radical biology for medicine: learning from nonalcoholic fatty liver disease. Free Radic Biol Med 2013;65:952-68. doi:10.1016/j.freeradbiomed.2013.08.174.

[25] Tilg H, Moschen AR. Evolution of inflammation in nonalcoholic fatty liver disease: The multiple parallel hits hypothesis. Hepatology 2010;52:1836-46. doi:10.1002/hep.24001.

[26] Rector RS, Thyfault JP, Uptergrove GM, Morris EM, Naples SP, Borengasser SJ, et al. Mitochondrial dysfunction precedes insulin resistance and hepatic steatosis and contributes to the natural history of non-alcoholic fatty liver disease in an obese rodent model. J Hepatol 2010;52:727-36. doi:10.1016/j.jhep.2009.11.030.

[27] Bournat JC, Brown CW. Mitochondrial dysfunction in obesity. Curr Opin Endocrinol Diabetes Obes 2010;17:446-52. doi:10.1097/MED.0b013e32833c3026.

[28] Petersen KF, Befroy D, Dufour S, Dziura J, Ariyan C, Rothman DL, et al. Mitochondrial dysfunction in the elderly: possible role in insulin resistance. Science 2003;300:1140-2. doi:10.1126/science.1082889.

[29] García-Ruiz C, Colell A, Morales A, Kaplowitz N, Fernández-Checa JC. Role of oxidative stress generated from the mitochondrial electron transport chain and mitochondrial glutathione status in loss of mitochondrial function and activation of transcription factor nuclear factor-kappa B: studies with isolated mitochondria and rat hepatocytes. Mol Pharmacol 1995;48:825-34.

[30] Vander Heiden MG, Chandel NS, Williamson EK, Schumacker PT, Thompson CB. BclxL Regulates the Membrane Potential and Volume Homeostasis of Mitochondria. Cell 1997;91:627-37. doi:10.1016/S0092-8674(00)80450-X.

[31] Kučera O, Garnol T, Lotková H, Staňková P, Mazurová Y, Hroch M, et al. The effect of rat strain, diet composition and feeding period on the development of a nutritional 
model of non-alcoholic fatty liver disease in rats. Physiol Res Acad Sci Bohemoslov 2011;60:317-28.

[32] Desvergne B, Michalik L, Wahli W. Transcriptional Regulation of Metabolism. Physiol Rev 2006;86:465-514. doi:10.1152/physrev.00025.2005.

[33] Jin J, Iakova P, Breaux M, Sullivan E, Jawanmardi N, Chen D, et al. Increased expression of enzymes of triglyceride synthesis is essential for the development of hepatic steatosis. Cell Rep 2013;3:831-43. doi:10.1016/j.celrep.2013.02.009.

[34] Pettinelli P, Videla LA. Up-regulation of PPAR-gamma mRNA expression in the liver of obese patients: an additional reinforcing lipogenic mechanism to SREBP-1c induction. J Clin Endocrinol Metab 2011;96:1424-30. doi:10.1210/jc.2010-2129.

[35] Sekiya M, Hiraishi A, Touyama M, Sakamoto K. Oxidative stress induced lipid accumulation via SREBP1c activation in HepG2 cells. Biochem Biophys Res Commun 2008;375:602-7. doi:10.1016/j.bbrc.2008.08.068.

[36] Peterson J, Dwyer J, Bhagwat S, Haytowitz D, Holden J, Eldridge AL, et al. Major flavonoids in dry tea. $J$ Food Compos Anal 2005;18:487-501. doi:10.1016/j.jfca.2004.05.006.

[37] Zuo Y, Chen H, Deng Y. Simultaneous determination of catechins, caffeine and gallic acids in green, Oolong, black and pu-erh teas using HPLC with a photodiode array detector. Talanta 2002;57:307-16.

[38] Chung M-Y, Park HJ, Manautou JE, Koo SI, Bruno RS. Green tea extract protects against nonalcoholic steatohepatitis in ob/ob mice by decreasing oxidative and nitrative stress responses induced by proinflammatory enzymes. J Nutr Biochem 2012;23:361-7. doi:10.1016/j.jnutbio.2011.01.001.

[39] Frei B, Higdon JV. Antioxidant Activity of Tea Polyphenols In Vivo: Evidence from Animal Studies. J Nutr 2003;133:3275S-3284S.

[40] Kuzu N, Bahcecioglu IH, Dagli AF, Ozercan IH, Ustündag B, Sahin K. Epigallocatechin gallate attenuates experimental non-alcoholic steatohepatitis induced by high fat diet. J Gastroenterol Hepatol 2008;23:e465-70. doi:10.1111/j.14401746.2007.05052.x.

[41] Nakamoto K, Takayama F, Mankura M, Hidaka Y, Egashira T, Ogino T, et al. Beneficial Effects of Fermented Green Tea Extract in a Rat Model of Non-alcoholic Steatohepatitis. J Clin Biochem Nutr 2009;44:239-46. doi:10.3164/jcbn.08-256.

[42] Park HJ, Lee J-Y, Chung M-Y, Park Y-K, Bower AM, Koo SI, et al. Green tea extract suppresses NFKB activation and inflammatory responses in diet-induced obese rats with nonalcoholic steatohepatitis. J Nutr 2012;142:57-63. doi:10.3945/jn.111.148544.

[43] Yesilova Z, Yaman H, Oktenli C, Ozcan A, Uygun A, Cakir E, et al. Systemic Markers of Lipid Peroxidation and Antioxidants in Patients with Nonalcoholic Fatty Liver Disease. Am J Gastroenterol 2005;100:850-5. doi:10.1111/j.1572-0241.2005.41500.x.

[44] Wang S, Moustaid-Moussa N, Chen L, Mo H, Shastri A, Su R, et al. Novel insights of dietary polyphenols and obesity. J Nutr Biochem 2014;25:1-18. doi:10.1016/j.jnutbio.2013.09.001.

[45] Wu L-Y, Juan C-C, Ho L-T, Hsu Y-P, Hwang LS. Effect of Green Tea Supplementation on Insulin Sensitivity in Sprague-Dawley Rats. J Agric Food Chem 2004;52:643-8. doi:10.1021/jf030365d.

[46] Fardet A, Chardigny J-M. Plant-Based Foods as a Source of Lipotropes for Human Nutrition: A Survey of In Vivo Studies. Crit Rev Food Sci Nutr 2013;53:535-90. doi:10.1080/10408398.2010.549596.

[47] Millán J, Pintó X, Muñoz A, Zúñiga M, Rubiés-Prat J, Pallardo LF, et al. Lipoprotein ratios: Physiological significance and clinical usefulness in cardiovascular prevention. Vasc Health Risk Manag 2009;5:757-65. 
[48] Khosravi S, Alavian SM, Zare A, Daryani NE, Fereshtehnejad S-M, Daryani NE, et al. Non-alcoholic fatty liver disease and correlation of serum alanin aminotransferase level with histopathologic findings. Hepat Mon 2011;11:452-8.

[49] Balkau B, Vernay M, Mhamdi L, Novak M, Arondel D, Vol S, et al. The incidence and persistence of the NCEP (National Cholesterol Education Program) metabolic syndrome. The French D.E.S.I.R. study. Diabetes Metab 2003;29:526-32. doi:10.1016/S1262-3636(07)70067-8.

[50] Sanyal D, Mukherjee P, Raychaudhuri M, Ghosh S, Mukherjee S, Chowdhury S. Profile of liver enzymes in non-alcoholic fatty liver disease in patients with impaired glucose tolerance and newly detected untreated type 2 diabetes. Indian J Endocrinol Metab 2015;19:597-601. doi:10.4103/2230-8210.163172. 


\section{Tables}

Table 1. Experimental diets composition.

\begin{tabular}{lccc}
\hline Composition & Control & HFS & HFS + Tea \\
\hline Lipids (\%) & 3.1 & 35.8 & 35.8 \\
Proteins (\%) & 16.1 & 17.4 & 17.4 \\
Carbohydrates (\%) & 3.37 & 35 & 35 \\
Crude fibers (\%) & 3.9 & 0 & 0 \\
Minerals (\%) & 4.5 & 5.8 & 5.8 \\
NFE (\%) & 59.9 & 33.3 & 33.3 \\
Vitamin A (UI/kg) & 6600 & 20000 & 20000 \\
Vitamin D3 (UI/kg) & 900 & 2500 & 2500 \\
Vitamin E (UI/kg) & 30 & 175 & 175 \\
\hline
\end{tabular}

NFE $($ Nitrogen free extract $)=100-\left(\mathrm{H}_{2} \mathrm{O}\right)-($ Protein $)-($ fat $)-($ Mineral $)-($ crude fiber $)$

Table 2. Total polyphenol, total catechin, gallic acid and caffeine content $(\mathrm{mg} / 100 \mathrm{ml})$ of tea infusion given to the rats.

\begin{tabular}{lc}
\hline Compound structures & $\mathbf{m g} / \mathbf{1 0 0} \mathbf{~ m l ~ o f ~ a q u e o u s ~ i n f u s i o n}{ }^{\mathbf{1}}$ \\
\hline Total polyphenol & $126.6 \pm 0.63$ \\
Total catechin & $10.7 \pm 0.56$ \\
Epigallocatechin & $1.2 \pm 0.13$ \\
Epicatechin & $2.2 \pm 0.13$ \\
Epigallocatechin gallate & $5.5 \pm 0.19$ \\
Epicatechin gallate & $1.8 \pm 0.13$ \\
Gallic acid (GA) & $8.4 \pm 0.19$ \\
Caffeine & $41.12 \pm 0.31$ \\
\hline
\end{tabular}

${ }^{1}$ Results are expressed as means \pm SEM of three parallel measurements $(n=3)$.

The tea infusions were prepared by pouring $200 \mathrm{~mL}$ of distilled water at $100{ }^{\circ} \mathrm{C}$ on $20 \mathrm{~g}$ of tea leaves mixture powder and brewing for $15 \mathrm{~min}$. The given tea infusion obtained was then diluted in 4 volumes of tap water. 


\section{Figures}

Figure 1. Effects of tea supplementation on obesity and associated risk factors in Wistar rats. Animals were sacrificed after 14 weeks on a standard diet (control) or on a high fatsucrose diet (HFS) diet. Sucrose (10\%) was supplied in the drink for HFS groups and tea infusion was supplied as drink for HFS+Tea group. (A) Changes in body weight and fat mass, (B) fasting glucose (left panel) and oral glucose tolerance test (right panel) and (C) Assessment of triglycerides (first left panel), non esterified fatty acid (NEFA, second left panel), low density lipoprotein (LDL, middle panel), high density lipoprotein (HDL, fourth left panel) and ratio LDL/HDL (right panel) in plasma. The values represent means \pm SEM, Control $(\mathrm{n}=13-14)$, HFS $(\mathrm{n}=13-15)$ and HFS+Tea $(\mathrm{n}=13-15) .{ }^{*} p<0.05$ compared with the control group and ${ }^{\#} p<0.05$ compared with the HFS group.

Figure 2. Tea prevents hepatic lipid accumulation induced by HFS diet in Wistar rats.

(A) Macroscopic pictures and Oil Red O stained liver from control, HFS and HFS+Tea rats,

(B) Liver weight (g), (C) Total lipid content, (D) Non-esterified fatty acid (NEFA) content,

(E) Triglycerides content, (F) Total cholesterol content, and (G) Plasma level of alanine transaminase (ALT). The values represent means \pm SEM, Control $(n=8)$, HFS $(n=15)$ and HFS+Tea $(\mathrm{n}=15) .{ }^{*} p<0.05$ compared with the control group and ${ }^{*} p<0.05$ compared with the HFS group.

Figure 3. Tea attenuates liver and plasma oxidative stress. (A) Hepatic lipid peroxidation as malondialdehyde (MDA) equivalent, (B) Hepatic total antioxidant capacity as trolox equivalent, (C) Plasma total antioxidant capacity and (D) Correlation between hepatic lipid peroxidation and hepatic total lipid content. The values represent means \pm SEM, Control $(\mathrm{n}=8-10)$, HFS $(\mathrm{n}=8-10)$ and HFS+Tea $(\mathrm{n}=8-10) .{ }^{*} p<0.05$ compared with the control group and ${ }^{\#} p<0.05$ compared with the HFS group.

Figure 4. Tea inhibits oxidative stress and lipid accumulation induced by pro-oxidant $t$ BHP treatment in a primary culture of rat hepatocytes. Cells were seeded onto 96-well Eplates, treated for $12 \mathrm{~h}$ with or without t-BHP $(120 \mu \mathrm{M})$ in presence (pre-treated) or not (nonpretreated) of various concentrations of tea extracts $(100,250$ and $500 \mu \mathrm{g} / \mathrm{mL})$ or tempol $(50 \mu \mathrm{M})$. Cells were labeled with Hoechst 33342 and with (A) MitoSox or (B) LipidTox for 
30 min before being read on the ArrayScanXTI. (A) Mitochondrial superoxide $\left(\mathrm{O}_{2}{ }^{\circ}\right)$ content and (B) Lipid accumulation were calculated from the MitoSox and LipidTox fluorescence intensity respectively. (C) Correlation between MitoSox staining and LipidTox staining in primary culture of rat hepatocytes. Error bars indicate the mean \pm SEM of triplicate determinations in three independent experiments (note: ${ }^{*} p<0.05$ when compared to nontreated cells, $\stackrel{\#}{p}<0.05$ when compared to non-pretreated $+\mathrm{t}-\mathrm{BHP}$ treated cells). Objective magnification x 20 .

Figure 5. Schematic illustration of potential anti-lipogenesis properties of tea through its antioxidant activities. $\rightarrow$ Activation or increase; $\perp$ or x Inhibition or decrease; 
(A)
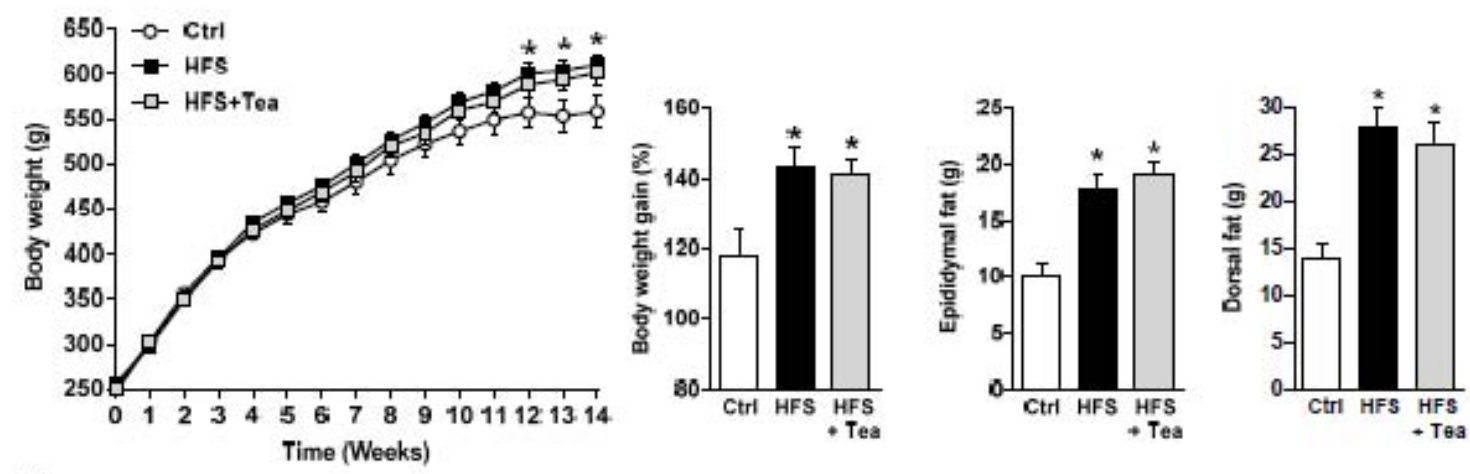

(B)
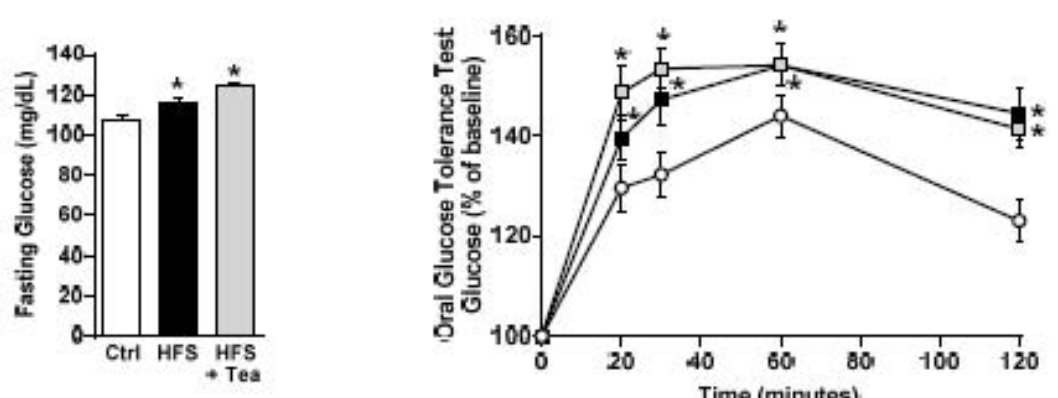

$-0-C$ tri

- HFS

- - HFS+Tea

(c)
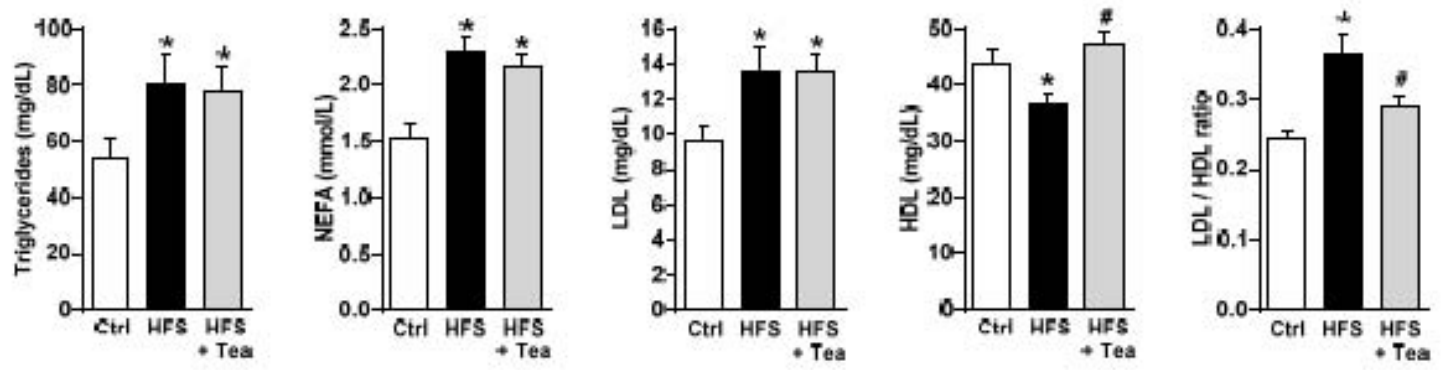

Figure 1 
(A)

Control

HFS

HFS+Tea

Macroscopic pictures

Oil Red $O$ staining

$\underline{\mathrm{ma} \mathrm{N}_{\mathrm{M}} \mathrm{M}}$
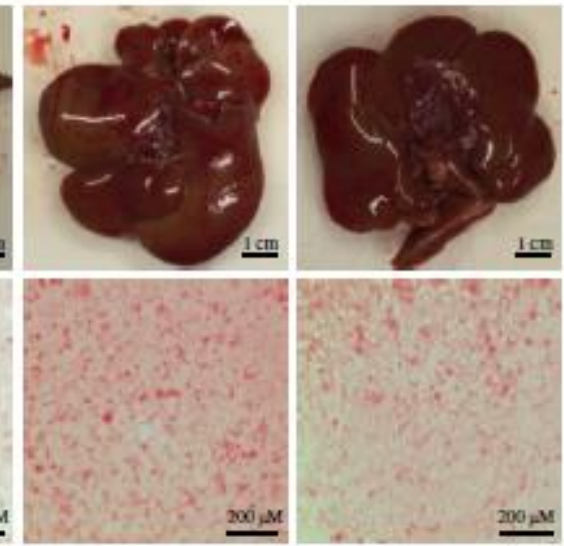

(B)

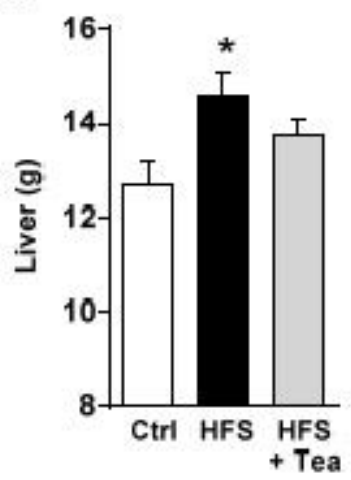

(E)

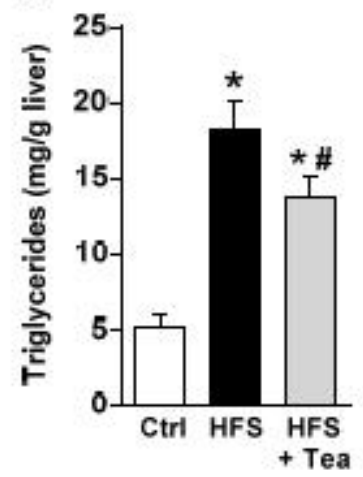

(C)

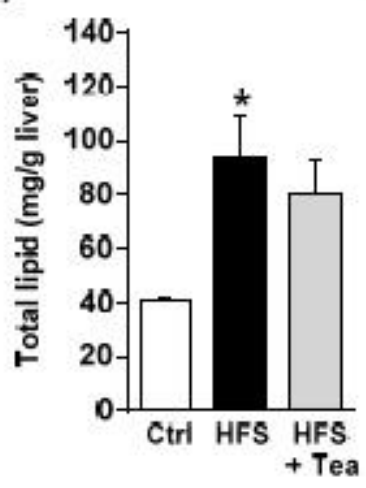

(F)

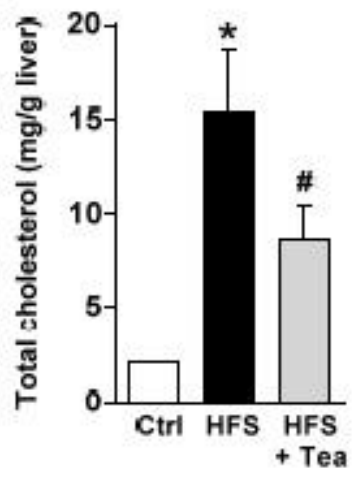

(D)

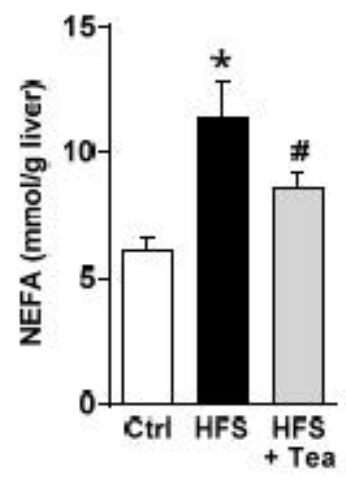

(G)

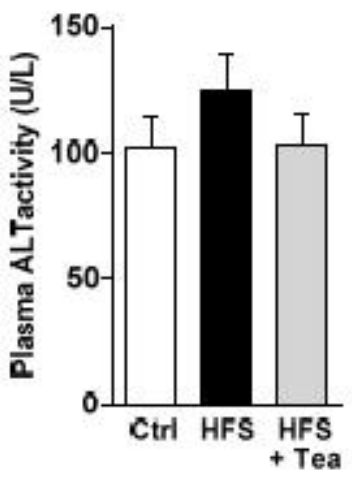

Figure 2 
(A)

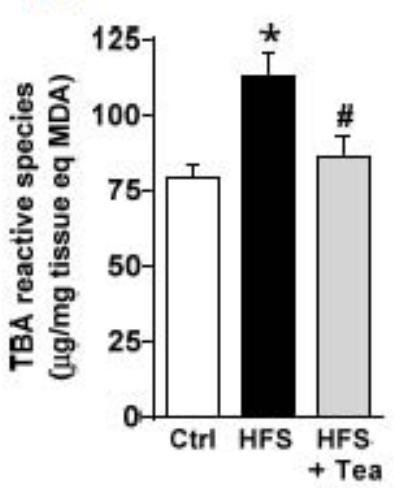

(B)

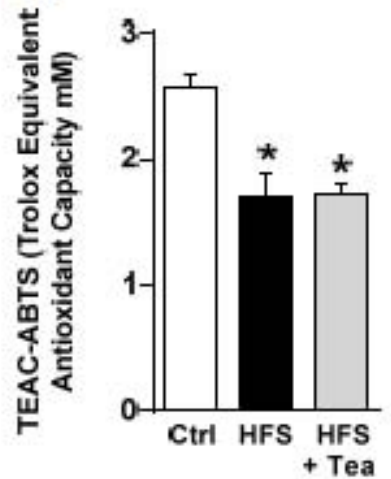

(C)

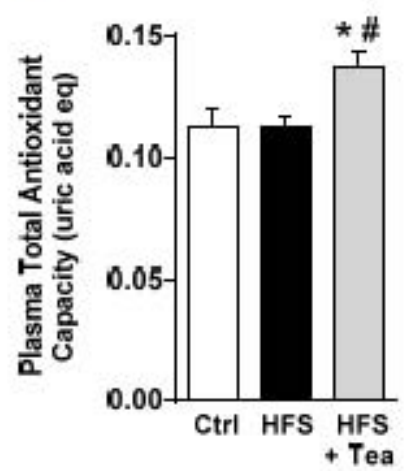

(D) Correlation between hepatic lipid peroxidation

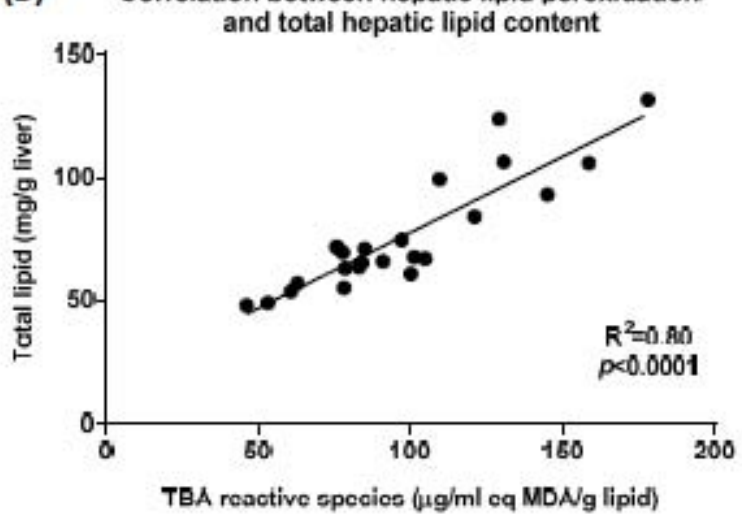

Figure 3 
(A)

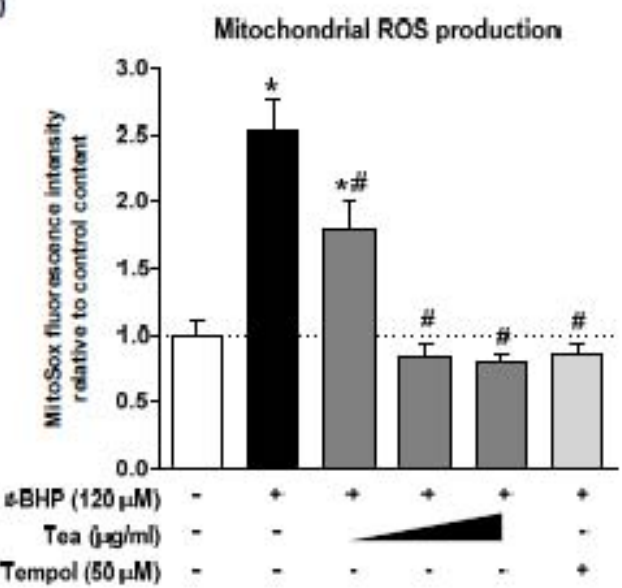

(B)

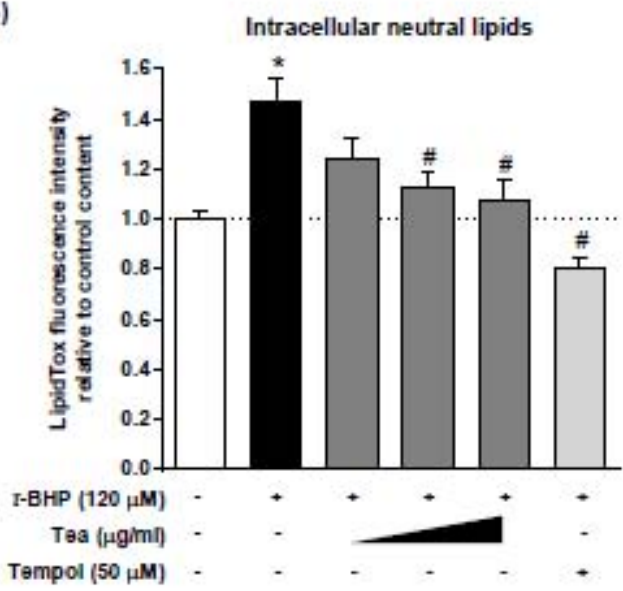

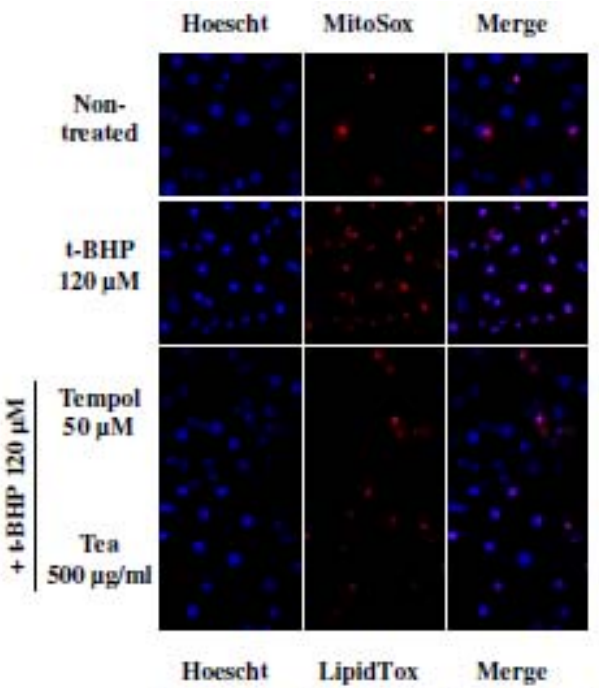

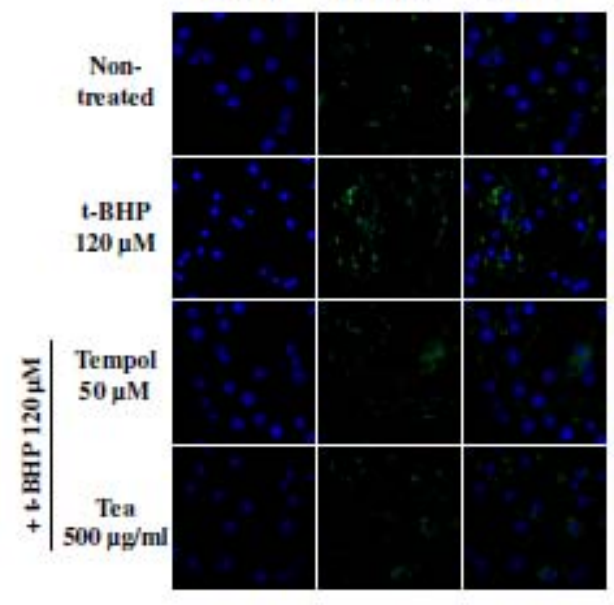

(c) Correlation between Mitosox and LipidTox stainings

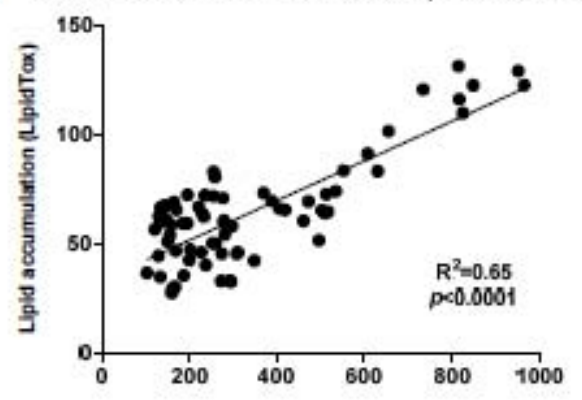

Figure 4 


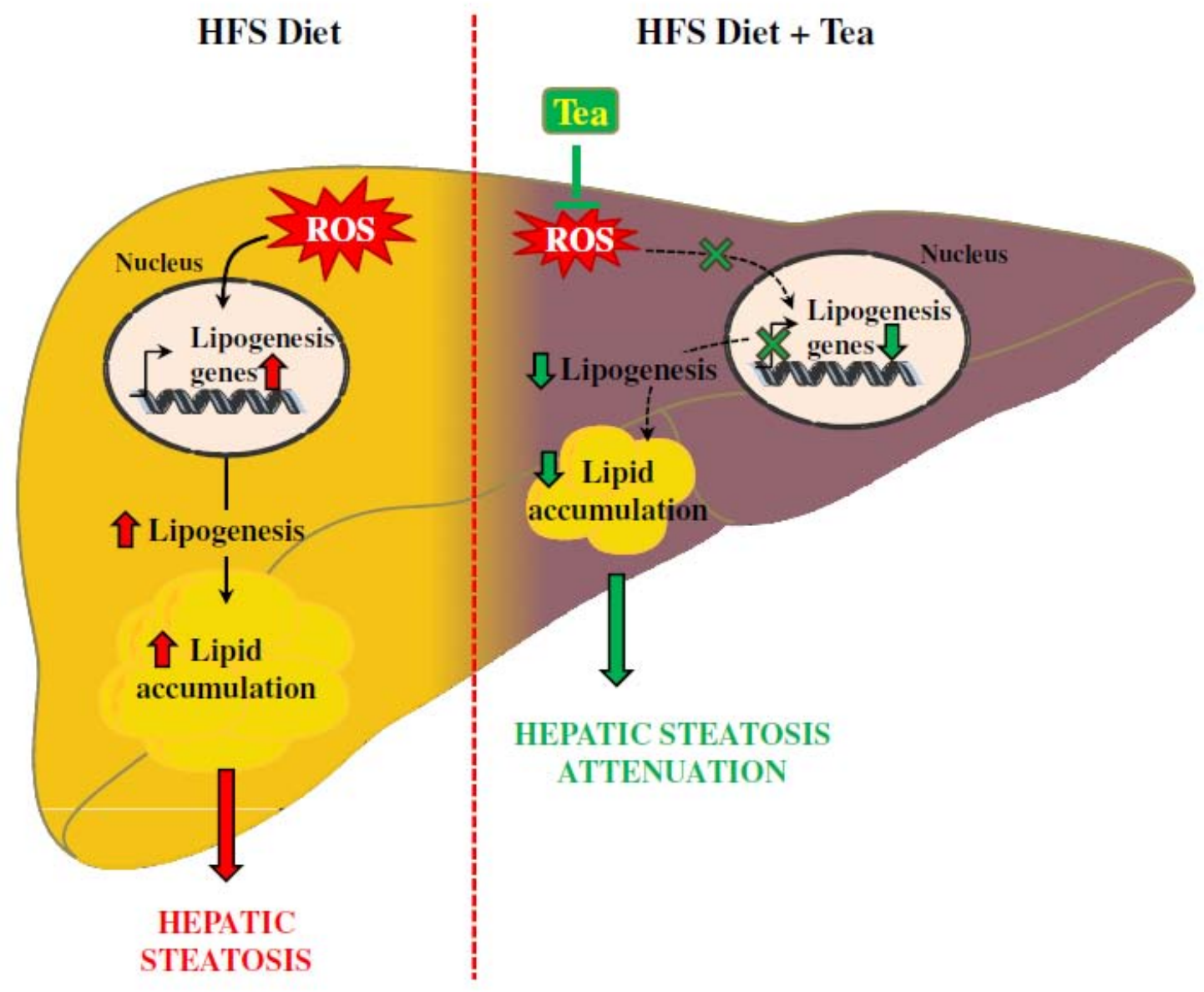

Figure 5 


\section{Highlights}

- HFS-diet induced hepatic steatosis in rats is prevented by tea

- Tea reduces HFS diet-induced oxidative stress in the liver

- Increased ROS production in hepatocytes results in lipid accumulation

- ROS-dependent lipid accumulation in hepatocytes is blunted by both tempol and tea 